\title{
Bright x-ray radiation from plasma bubbles in an evolving laser wakefield accelerator
}

\author{
M. S. Bloom, ${ }^{1}$ M. J. V. Streeter $\odot,{ }^{1,}{ }^{*}$ S. Kneip,${ }^{1}$ R. A. Bendoyro, ${ }^{2}$ O. Cheklov, ${ }^{3}$ J. M. Cole $\odot,{ }^{1}$ \\ A. Döpp, ${ }^{1,4,5}$ C. J. Hooker, ${ }^{3}$ J. Holloway, ${ }^{6}$ J. Jiang, ${ }^{2}$ N. C. Lopes,${ }^{1,2}$ H. Nakamura, ${ }^{1}$ \\ P. A. Norreys, ${ }^{3}$ P. P. Rajeev, ${ }^{3}$ D. R. Symes, ${ }^{3}$ J. Schreiber, ${ }^{1,4}$ J. C. Wood, ${ }^{1}$ M. Wing $\odot,{ }^{6}$ \\ Z. Najmudin $\odot{ }^{1}$ and S. P. D. Mangles $\oplus^{1, \dagger}$ \\ ${ }^{1}$ The John Adams Institute for Accelerator Science, Imperial College London, \\ London, SW7 2AZ, United Kingdom \\ ${ }^{2}$ GoLP/Instituto de Plasmas e Fusão Nuclear, Instituto Superior Técnico, U.L., Lisboa 1049-001, Portugal \\ ${ }^{3}$ Central Laser Facility, STFC Rutherford Appleton Laboratory, Didcot OX11 OQX, United Kingdom \\ ${ }^{4}$ Fakultät für Physik, Ludwig-Maximilians-Universität München, D-85748 Garching, Germany \\ ${ }^{5}$ Max-Planck-Institut für Quantenoptik, Hans-Kopfermann-Strasse 1, D-85748 Garching, Germany \\ ${ }^{6}$ Department of Physics and Astronomy, University College London, London WCIE 6BT, United Kingdom
}

(Received 6 November 2017; revised manuscript received 23 October 2019; accepted 6 May 2020; published 26 June 2020)

\begin{abstract}
We show that the properties of the electron beam and bright $\mathrm{x}$ rays produced by a laser wakefield accelerator can be predicted if the distance over which the laser self-focuses and compresses prior to self-injection is taken into account. A model based on oscillations of the beam inside a plasma bubble shows that performance is optimized when the plasma length is matched to the laser depletion length. With a $200 \mathrm{TW}$ laser pulse, this results in an x-ray beam with a median photon energy of $20 \mathrm{keV}$, $>6 \times 10^{8}$ photons above $1 \mathrm{keV}$ per shot, and a peak brightness of $3 \times 10^{22}$ photons s $\mathrm{mrad}^{-2} \mathrm{~mm}^{-2}$ $(0.1 \% \mathrm{BW})^{-1}$.
\end{abstract}

DOI: $10.1103 /$ PhysRevAccelBeams.23.061301

Laser wakefield accelerators [1] have gathered increasing interest since it was first shown that they were capable of producing high-quality electron beams [2-4]. Development has continued apace, and laser wakefield accelerators can now produce ultrashort bunches of electrons, down to a few femtoseconds [5], and reach multi$\mathrm{GeV}$ beam energies [6-8]. One of the primary near-term uses of laser wakefield accelerators is the production of bright, femtosecond-duration pulses of broadband $\mathrm{x}$ rays $[9,10]$, that are suitable for a range of applications [11].

A laser wakefield accelerator is formed when an intense, short-duration laser pulse is fired into a moderate-density plasma. The ponderomotive force associated with the laser pushes plasma electrons out of its way as it propagates. The much heavier positive ions are effectively stationary, and so the electrons are pulled back toward their equilibrium positions once the laser has passed. The resulting collective charge oscillation has a relativistic phase velocity in the

\footnotetext{
*m.streeter09@imperial.ac.uk

stuart.mangles@imperial.ac.uk
}

Published by the American Physical Society under the terms of the Creative Commons Attribution 4.0 International license. Further distribution of this work must maintain attribution to the author(s) and the published article's title, journal citation, and DOI. wake of the laser. When driven by a sufficiently intense laser pulse, almost all of the electrons can be expelled from an approximately spherical cavity behind the drive, known as the plasma bubble [12]. The electric fields inside this bubble are capable of accelerating electrons to $\sim 1 \mathrm{GeV}$ in just $\sim 1 \mathrm{~cm}[13]$.

Electrons can be self-injected into the bubble from the background plasma if the wave has a sufficiently high amplitude [14]. The three-dimensional structure of the bubble means that the injected electron beam undergoes strongly nonlinear betatron oscillations with a short wavelength $(\sim 1 \mathrm{~mm}$ for $\sim 1 \mathrm{GeV})$ and so generates synchrotron $\mathrm{x}$ rays in the multi-keV spectral range [9].

In this paper, we report on the experimental optimisation of the $\mathrm{x}$ rays generated by a laser wakefield accelerator driven by a 200 TW laser. We show that the electron and $\mathrm{X}$-ray properties are well described by an analytical model that includes the fact that self-injection occurs only after the pulse has self-focused and self-compressed to a sufficiently high intensity. We also show that this source outperforms previous reports of $\mathrm{x}$-ray emission from wakefield accelerators $[7,10]$.

The experiment was performed using the Astra Gemini laser at the Rutherford Appleton Laboratory, which delivered pulses of $12 \mathrm{~J}$ and duration $55 \mathrm{fs}$ FWHM onto a supersonic gas jet target. The laser was focused with an $f / 20$ off-axis parabolic mirror to a $22 \mu \mathrm{m}$ FWHM spot containing 


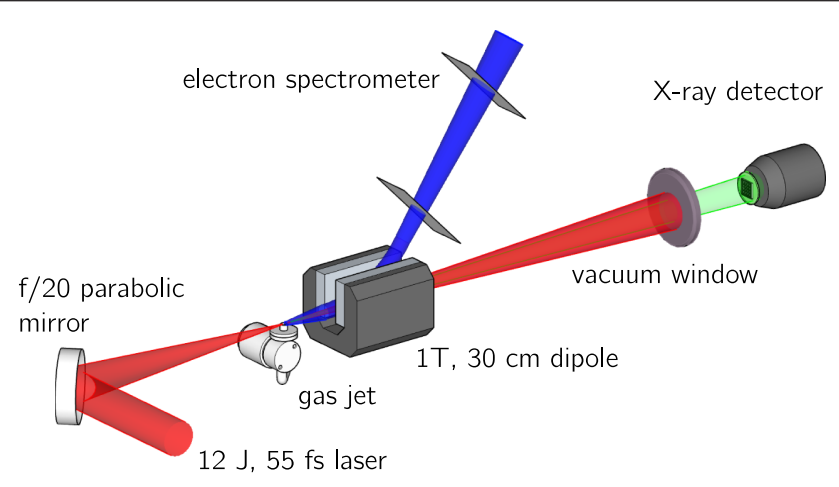

FIG. 1. Schematic of the experimental setup. A $12 \mathrm{~J}, 55 \mathrm{fs}$ laser pulse is focused by an $f / 20$ parabolic mirror onto a gas jet target which produces a centimeter-scale plasma with densities $\sim 10^{19} \mathrm{~cm}^{-3}$. Giga-electron-volt-scale electrons produced in the interaction are deflected by a dipole magnet onto two scintillating screens to measure the energy spectrum. Kilo-electron-volt x rays pass out of the vacuum chamber through a thin window and are detected by an indirect-detection x-ray CCD camera. An array of metallic filters in front of the CCD allows properties of the x-ray spectrum to be inferred.

$\alpha \approx 30 \%$ of the energy. The peak intensity at focus in vacuum was $I \approx 2.2 \times 10^{19} \mathrm{~W} \mathrm{~cm}^{-2}$, corresponding to a normalized vector potential $a_{0}=e A_{0} /\left(m_{\mathrm{e}} c^{2}\right) \simeq 3.0$.

Two different helium gas jet targets with exit diameters of $(10,15) \mathrm{mm}$ were used, producing approximately uniform density plasmas of length $(8.5,13) \mathrm{mm}$ with electron densities up to $(8.0,4.0) \times 10^{18} \mathrm{~cm}^{-3}$, respectively. The laser was focused onto the front edge of the gas flow. The generated electron beam was analyzed using a magnetic spectrometer consisting of a 30-cm-long $1 \mathrm{~T}$ permanent dipole magnet and two scintillating (Kodak Lanex regular) screens.

The magnet also swept the electron beam away from an indirect-detection $\mathrm{x}$-ray $\mathrm{CCD}$ camera (Princeton Instruments PIXIS) placed on the laser axis. This was mounted outside the vacuum chamber behind a $180-\mu \mathrm{m}-$ thick Be window. An array of 16 metallic filters mounted on a thin Al/Mylar substrate was placed directly in front of the camera's CsI scintillator. A schematic of the experimental layout is shown in Fig. 1.

The $\mathrm{x}$-ray spectrum was found by performing a least-squares fit to the signal detected behind each filter, taking into account the transmission through each filter and the absolutely calibrated detector response under the assumptions that the spectrum has a synchrotronlike shape given by $d^{2} I /(d E d \Omega)_{\theta=0} \propto \xi^{2} \mathcal{K}_{2 / 3}^{2}(\xi / 2)$, where $\mathcal{K}_{2 / 3}(x)$ is a modified Bessel function of the order of $2 / 3$ and $\xi=E / E_{\mathrm{c}}$. The shape of this spectrum is characterized by a single parameter, the critical energy $E_{\mathrm{c}}$ [15], which was assumed to be constant over the detector. Gaps between the $\mathrm{x}$-ray filters and repeated filters allowed gradients in the $\mathrm{x}$-ray beam profile to be taken into account.

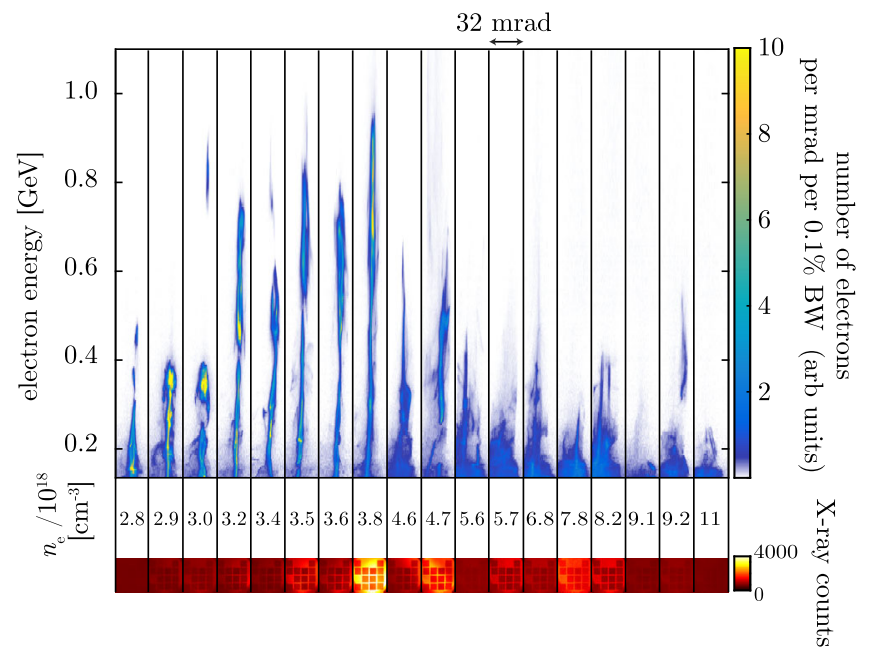

FIG. 2. Variation of electron and x-ray beams as a function of $n_{\mathrm{e}}$ for the $10 \mathrm{~mm}$ nozzle. Top: Dispersion-corrected spectrally dispersed images of the electron beam for a selection of shots. The horizontal axis represents the angle at which electrons exit the accelerator in the nondispersion plane. Bottom: X-ray CCD camera images for the same shots.

Figure 2 shows the variation of the electron beam spectrum and corresponding $\mathrm{x}$-ray $\mathrm{CCD}$ images with plasma density for the $10 \mathrm{~mm}$ nozzle. Shots in this data subset correspond to the brightest $\mathrm{x}$-ray image recorded at each density. The data show that there is an optimum density for acceleration of $n_{\mathrm{e}} \approx 3.8 \times 10^{18} \mathrm{~cm}^{-3}$ and that the $\mathrm{x}$-ray signal is correlated with the electron beam energy. Above the optimum density, the electron beam begins to develop transverse structure and increased divergence. This is consistent with the electron beam interacting with the plasma, driving its own wake, and becoming susceptible to propagation instabilities [19]. As the electron beam dephases, it can also interact with the laser field [20], which can also increase the x-ray flux [21]. However, under these conditions, no major enhancement in $\mathrm{x}$-ray flux is evident due to these effects.

Figure 3 shows the variation of the electron energy $W_{\max }$ with $n_{\mathrm{e}}$ for both the 10 and $15 \mathrm{~mm}$ gas nozzles. Both datasets show a similar trend; above a threshold density, $W_{\max }$ rapidly increases until it reaches a maximum, after which it decreases approximately $\propto 1 / n_{\mathrm{e}}$. Both the threshold and optimum densities occur at lower densities for the $15 \mathrm{~mm}$ nozzle. As the x-ray signal is optimized when the electron beam energy is highest, optimization of the $\mathrm{x}$-ray generation relies on understanding how to optimize the electron energy. The maximum energy predicted by Lu et al. [22],

$$
W_{\max } /\left(m_{\mathrm{e}} c^{2}\right)=\frac{2}{3} a_{0}\left(n_{\mathrm{c}} / n_{\mathrm{e}}\right),
$$

is plotted in Fig. 3 (dot-dashed line) using the $a_{0}$ for vacuum. Here, $n_{\mathrm{c}}=\epsilon_{0} m_{\mathrm{e}} \omega^{2} / e^{2}$ is the critical plasma density for 


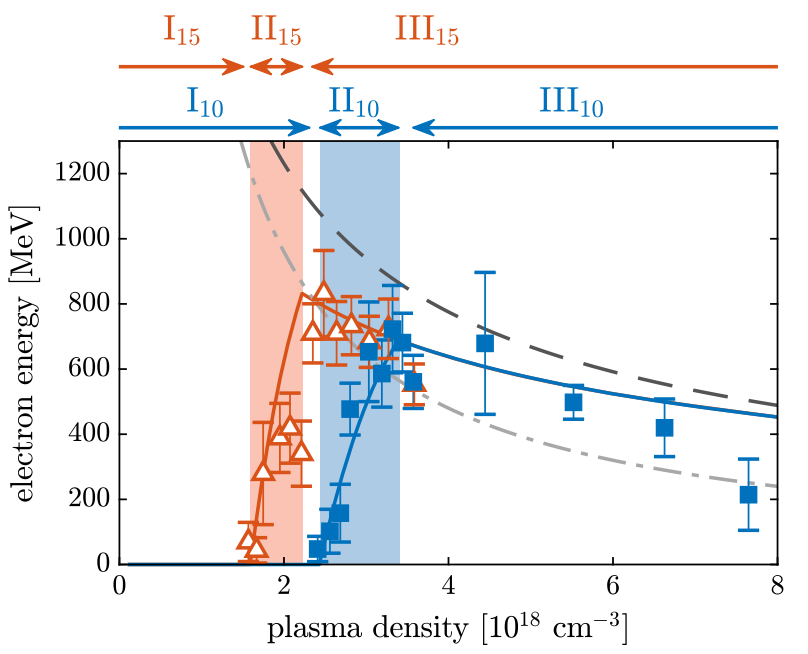

FIG. 3. Variation of $W_{\max }$ with $n_{\mathrm{e}}$ for 10 (squares) and $15 \mathrm{~mm}$ (triangles) gas jets. Each point is the mean from $N=1-13$ shots, and bars represent the combined statistical and measurement errors. Curves show the maximum energy predicted using Eqs. (1) (dot-dashed), (3) (dashed), and (4) for the 10 (blue) and $15 \mathrm{~mm}$ (red) nozzles. The regions (I, II, and III) described in the text are indicated for each nozzle. Region II for each nozzle is also indicated as a shaded region.

radiation with angular frequency $\omega$. Equation (1) consistently underestimates the electron energy at high densities and does not predict an optimum density.

Equation (1) assumes a nonevolving laser pulse. However, self-focusing and pulse compression occur as the pulse propagates in its self-generated wakefield, causing $a_{0}$ to increase. The maximum value of $a_{0}$ can be calculated by assuming that the pulse evolves to a matched spot size that satisfies $a_{0} \approx 2\left(P_{\mathrm{f}} / P_{\mathrm{c}}\right)^{1 / 3}$ [22], where $P_{\mathrm{c}}=$ $\left(2 m_{\mathrm{e}} c^{3} / r_{\mathrm{e}}\right) n_{\mathrm{c}} / n_{\mathrm{e}}$ is the critical power for relativistic selffocusing and $r_{\mathrm{e}}$ is the classical electron radius. The final power $P_{\mathrm{f}}$ depends on pulse compression and the amount of laser energy that is trapped in the bubble. For a fraction $\alpha$ of the total laser energy $E_{\mathrm{L}}$ compressed to a pulse duration $\tau_{\mathrm{f}}$, the resulting expression is

$$
a_{\max } \simeq 2\left[\alpha E_{\mathrm{L}} /\left(\tau_{\mathrm{f}} P_{\mathrm{c}}\right)\right]^{1 / 3} .
$$

Even though this expression does not include the effects of pump depletion and photon deceleration, it has been shown to be sufficiently accurate to predict the selfinjection threshold [14]. The pulse compression can be quantified by assuming that the front of the pulse travels at the linear group velocity in the plasma, while the rear of the pulse, which sits in the significantly reduced plasma density inside the bubble, travels at $c$. For an initial pulse duration $\tau_{0}$, the pulse duration after propagation length $l$ is then $\tau_{\mathrm{f}}(l) \approx \tau_{0}-n_{\mathrm{e}} l /\left(2 c n_{\mathrm{c}}\right)$ [23]. The maximum propagation length $L_{\max }$ will be limited by pump depletion $L_{\mathrm{pump}} \approx$ $c \tau_{0} n_{\mathrm{c}} / n_{\mathrm{e}}$ [22] or by the length of the target, $L_{\text {target }}$, if
$L_{\text {target }}<L_{\text {pump. }}$. So, accounting for pulse evolution, the beam energy varies as

$$
W_{\max }^{\prime} /\left(m_{\mathrm{e}} c^{2}\right) \approx \frac{4}{3}\left[\alpha E_{\mathrm{L}} /\left(\tau_{\mathrm{f}} P_{\mathrm{c}}\right)\right]^{1 / 3}\left(n_{\mathrm{c}} / n_{\mathrm{e}}\right) .
$$

Equation (3) is shown as the dashed line in Fig. 3. This model overestimates the observed energy gain, approaching the data only at high densities. It also still fails to explain the initial increase in beam energy with increasing density. These features can be explained by including a distance over which the $a_{0}$ amplification occurs before self-injection. We call this the preinjection pulse evolution (PIPE) length $L_{\text {PIPE }}$. This PIPE length will decrease at higher densities as the pulse evolution rates increase [24]. The variation of electron energy with density can be split into three regions. At low density (region I), the PIPE length is longer than the gas jet, and so no electrons are injected.

As the density is increased, the evolution becomes fast enough that injection occurs before the end of the gas jet, resulting in low-energy electron beams. This region II is marked by the shaded area in Fig. 3. Increasing the density further reduces the PIPE length and brings the injection point earlier in the gas jet. But in region II, the density is low enough that the laser has not depleted by the end of the gas jet, so earlier injection leads to an increase in acceleration length. This, coupled with the increase in the accelerating field $\left(\propto \sqrt{n_{\mathrm{e}}}\right)$, results in a rapid increase of beam energy with increasing density.

Once the density is high enough that the pump depletion length is less than the gas jet length (region III), increasing the density actually decreases the length over which the electron beam is accelerated. Therefore, in region III, despite the continuing increase in electric field strength, higher density results in a decrease in the electron energy. At the highest densities, the PIPE length is very short, and the electron beam energy approaches the dephasing limit [Eq. (3)]. To model all this behavior, we need an expression for the energy gained by an electron accelerated over some fraction of a dephasing length and an expression that tells us what fraction of a dephasing length is available after the PIPE length is taken into account.

In the bubble regime, the longitudinal electric field, which is responsible for acceleration, is linearly proportional to the longitudinal distance from the bubble center, i.e., $E_{z}(\xi) \propto \xi$, where $\xi=z-v_{\phi} t$ and $v_{\phi}$ is the velocity of the bubble [25]. Because injected electrons travel faster than the bubble, they experience a decreasing accelerating electric field as they advance relative to the bubble. The energy gained by the electron is found by integrating the accelerating force over the path of the electron. To first order, $\mathrm{d} \xi / \mathrm{d} z \approx\left(c-v_{\phi}\right) / c$, and so the electron energy is quadratic with acceleration distance [26]. The maximum energy is reached when an electron travels from the back to 
the center of the bubble, i.e., when $\Delta \xi=r_{b} / 2$, which occurs when the acceleration length is equal to the dephasing length, $L_{\phi}=\frac{4}{3} a_{0}^{1 / 2}\left(n_{\mathrm{c}} / n_{\mathrm{e}}\right) c / \omega_{\mathrm{p}}$. Because injection occurs after the PIPE length, the fraction of the dephasing length available for acceleration is therefore $\Delta_{\text {acc }}=\left(L_{\max }-L_{\mathrm{PIPE}}\right) / L_{\phi}$, where $L_{\max }$ is the maximum length available for the laser wakefield accelerator: the shorter of $L_{\text {target }}$ and $L_{\text {pump }}$. The effect of the preinjection evolution phase is therefore to reduce the maximum energy reached compared to Eq. (3) according to

$$
W_{\max }^{\prime \prime}=W_{\max }^{\prime}\left(2 \Delta_{\mathrm{acc}}-\Delta_{\mathrm{acc}}^{2}\right) .
$$

Equation (4) is plotted in Fig. 3 (solid red and blue lines) with a single fitting parameter, $S=11.8 \pm 1.8 \mu \mathrm{m}$, chosen to best reproduce the experimental trend using a leastsquares fit. As self-focusing happens more quickly than pulse compression in this regime, the PIPE length is closely related to the length over which pulse amplification occurs, $L_{\text {evol }}$, which can be calculated using the model in Ref. [27]. That predicts that $S=\left(n_{e} / n_{c}\right) L_{\mathrm{evol}}=11 \mu \mathrm{m}$ for $n_{e}=$ $2 \times 10^{18} \mathrm{~cm}^{-3}$ with a weak dependence on plasma density. The PIPE model reproduces all of the main features of the experimentally observed variation in electron energy including the rapid rise to an optimum density, the reduction in optimum density for the longer nozzle, and the slower falloff of maximum energy at higher densities. The model performs equally well for both the 10 and $15 \mathrm{~mm}$ nozzles with the same value for $S$, indicating that the precise shape of the gas density plays a minor role in determining the evolution as compared to the initial laser parameters.

Perhaps counterintuitively, this shows that for our value of $S$ the maximum electron energy from a fixed length target occurs at too low a density for acceleration over a full dephasing length, i.e., $\Delta_{\text {acc }}<1$. Rather, the optimum occurs at the lowest density where the acceleration is limited by pump depletion, i.e., when $L_{\mathrm{PIPE}}+\Delta_{\mathrm{acc}} L_{\phi}=$ $L_{\text {target }}=L_{\text {pump. }}$. In general, for any value of $S$, the electron energy from a fixed length target is maximized for the density at which $L_{\text {target }}=L_{\text {pump }}$ or $L_{\text {target }}=L_{\phi}-L_{\mathrm{PIPE}}$, whichever occurs at a lower density. Increasing the length of the target allows for higher electron energies at a lower density (as shown by the $15 \mathrm{~mm}$ nozzle data), but this cannot be done indefinitely, as at some density the pulse will never evolve to the point of injection.

Figure 4 plots how the x-ray critical energy $E_{\mathrm{c}}$ and peak brightness $\mathcal{B}_{0}$ vary with $n_{\mathrm{e}}$ for the $10 \mathrm{~mm}$ gas nozzle. Both the critical energy and brightness show similar behavior, rapidly increasing as the density is increased before turning over above $n_{\mathrm{e}} \approx 3.8 \times 10^{18} \mathrm{~cm}^{-3}$. The critical energy reaches $E_{\mathrm{c}} \approx 30 \mathrm{keV}$. The peak brightness, calculated assuming a constant duration matching that of the laser pulse (55 fs) and an rms source radius

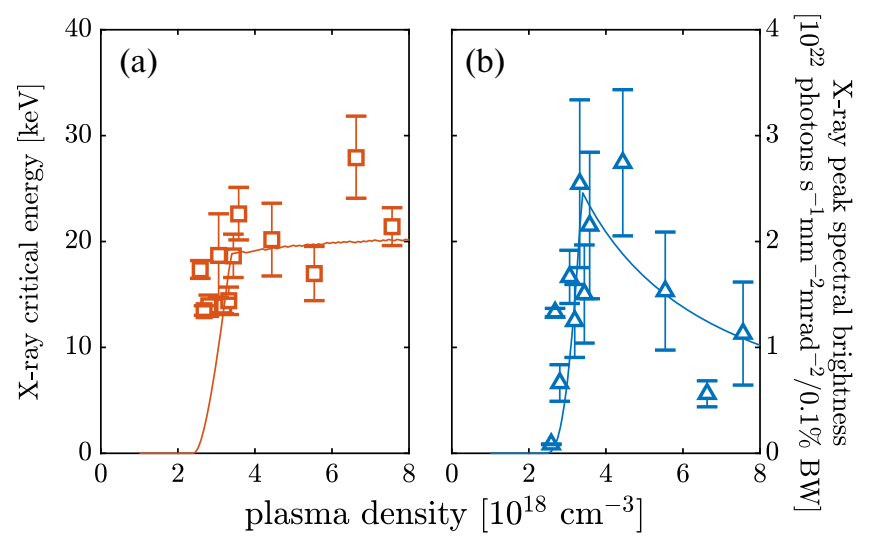

FIG. 4. Observed x ray: (a) $E_{\mathrm{c}}$ and (b) $\mathcal{B}_{0}$ as a function of $n_{\mathrm{e}}$ for the $10 \mathrm{~mm}$ nozzle. Each point is the mean of $N=1-10$ shots, and bars represent the combined statistical and measurement errors. Curves show predictions using Eqs. (5) and (6).

consistent with previous measurements $(1 \mu \mathrm{m})$, reaches $3 \times 10^{22}$ photons s${ }^{-1} \mathrm{mrad}^{-2} \mathrm{~mm}^{-2}(0.1 \% \mathrm{BW})^{-1}$. This is significantly higher than previous results at lower laser power (e.g., Ref. [10]), primarily due to the ability to accelerate electrons to $\sim \mathrm{GeV}$ energies, thus increasing their ability to radiate. Under optimum conditions, a total photon yield of $N_{\mathrm{X}} \approx 6 \times 10^{8}$ per shot above $1 \mathrm{keV}$ was measured.

By solving the equation of motion for an electron in the fields of a plasma bubble, Thomas [26] calculates the spectrum of radiation emitted by summing the synchrotron spectrum emitted at each bend in the trajectory as the electron is accelerated and then decelerated from the back of the bubble to the front, i.e., over a distance $L=2 L_{\phi}$. The number of betatron oscillations is found to be $N_{\beta}=\gamma_{\mathrm{p}}$, where $\gamma_{\mathrm{p}}$ is the Lorentz factor associated with the bubble motion. The resulting $\mathrm{x}$-ray spectrum is synchrotronlike with an enhanced high-energy tail. We modify the expression in Ref. [26] to take into account that, in general, the acceleration does not take place over a full dephasing length. The modified spectrum for a beam charge of $e N_{\mathrm{b}}$ is

$$
\frac{\mathrm{d}^{2} I}{\mathrm{~d} \omega \mathrm{d} \Omega}=\gamma_{\mathrm{p}} \frac{3 e^{2}}{\pi^{3} \epsilon_{0} c} N_{\mathrm{b}}\left(\frac{W_{\max }^{\prime}}{m_{\mathrm{e}} c^{2}}\right)^{2} \mathcal{A}\left(\frac{\omega}{2 \omega_{\mathrm{c} 0}}, \Delta_{\mathrm{acc}}\right),
$$

where $E_{\mathrm{c} 0}=\hbar \omega_{\mathrm{c} 0}=7.75 \times 10^{-4} a_{0}^{5 / 2}\left(n_{\mathrm{c}} / n_{\mathrm{e}}\right)^{9 / 8}$ is the critical energy of a synchrotron spectrum corresponding to that emitted by an electron at the maximum energy $W_{\max }^{\prime}$. The spectral shape function $\mathcal{A}\left(\xi, \Delta_{\text {acc }}\right)$ is

$$
\mathcal{A}=\xi^{2} \int_{-1}^{\Delta_{\text {acc }}-1}\left(1-x^{2}\right)^{-3 / 2} \mathcal{K}_{2 / 3}^{2}\left[\xi\left(1-x^{2}\right)^{-7 / 4}\right] \mathrm{d} x .
$$

The calculated variation in $E_{\mathrm{c}}$ and $\mathcal{B}_{0}$ have been overlaid on the experimental data in Figs. 4(a) and 4(b), for a plasma length of $8.5 \mathrm{~mm}$ with $L_{\mathrm{PIPE}}=S n_{\mathrm{c}} / n_{\mathrm{e}}$ and $S=11.8 \mu \mathrm{m}$. 


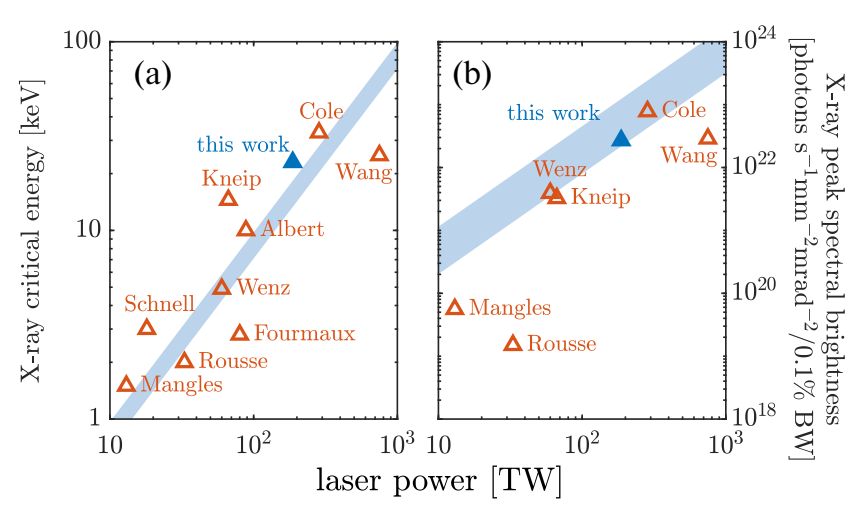

FIG. 5. Scaling of $\mathrm{x}$-ray radiation with laser power from various experiments [7,9,10,29-34]: (a) $E_{\mathrm{c}}$; (b) $\mathcal{B}_{0}$. The shaded region corresponds to Eqs. (7) and (8) for $n_{\text {th }} \leq n_{\mathrm{e}} \leq 3 n_{\text {th }}$.

The critical energy curve requires no additional fitting parameter beyond the PIPE length scaling already determined from the electron data. In the absence of an absolute charge calibration, we have used the scaling law of Lu et al. [22] multiplied by a fitting parameter $\hat{N}$, $N_{\mathrm{b}}=3.1 \times 10^{8} \hat{N} \lambda_{0} \sqrt{P_{\mathrm{f}}}$, in the calculation of peak brightness. Taking into account the effect of pulse compression, this gives an approximately constant charge of $\approx 44 \mathrm{pC}$ over the density range of interest. The peak brightness using this model is maximal at the density for which the pump depletion length equals the given target length. The models for the electron energy, x-ray critical energy, and density dependence of the x-ray brightness are all consistent with the experimental data using the single fitting parameter $S$.

Figure 5 compares our measured $E_{\mathrm{c}}$ and $\mathcal{B}_{0}$ with previous experiments. Kneip, Najmudin, and Thomas [28] calculated the following scaling laws in terms of the laser power $P$ (in TW) for both $E_{\mathrm{c}}$ (in $\mathrm{keV}$ ) and $\mathcal{B}_{0}$ [in photons $\mathrm{s}^{-1} \mathrm{mrad}^{-2} \mathrm{~mm}^{-2}(0.1 \% \mathrm{BW})^{-1}$ ]:

$$
\begin{gathered}
E_{\mathrm{c}}=3.5 \times 10^{-2}(\beta \alpha P)^{5 / 6}\left(n_{\mathrm{c}} / n_{\mathrm{e}}\right)^{7 / 24}, \\
\mathcal{B}_{0}=2.7 \times 10^{19} \hat{N}(\beta \alpha P)^{2 / 3}\left(n_{\mathrm{c}} / n_{\mathrm{e}}\right)^{19 / 12},
\end{gathered}
$$

where a power amplification factor $\beta$ is included to account for pulse compression. Compression over a depletion length at the rate in Ref. [23] gives $\beta=2$.

Equations (7) and (8) treat $P$ and $n_{\mathrm{e}}$ as independent parameters. However, increasing $P$ decreases the threshold density for self-injection, $n_{\text {th }}$, so experiments at higher power typically operate at lower density [35]. Using Ref. [14] to find $n_{\text {th }}(P)$, we can therefore eliminate $n_{\mathrm{e}}$ from the above expressions. Furthermore, experiments are typically optimized just above the threshold, so the curves for $E_{c}$ and $\mathcal{B}_{0}$ (plotted in Fig. 5) span the range $\left[n_{\mathrm{th}}, 3 n_{\mathrm{th}}\right]$.

The scaling laws and experimental data both show that higher-power lasers produce higher energy and brighter $\mathrm{x}$-ray sources. However, it is important to note that experiments can differ significantly from the scaling law. For example, higher photon energies can be produced if asymmetries are present in the wake, which increases the betatron oscillation amplitude. However, this also increases the source size, decreasing the brightness [29].

The photon source in our experiments is much brighter, at higher photon energy than those at lower power [10], and comparable to an unoptimized experiment at 5 times higher laser power [7]. Our experimental data and the model developed here suggest that a significant increase in the flux of x-ray radiation from a laser wakefield accelerator can be achieved by careful optimization of the length and plasma density for a given laser system. This would greatly broaden the range of applications that are accessible with these sources [11].

The authors confirm that all data used in this study are available without restriction. Data and analysis codes can be obtained using the link in Ref. [36].

The authors thank the staff at the Central Laser Facility, Rutherford Appleton Laboratory for their assistance. This research was supported by STFC (ST/J002062/1, ST/P002021/1), EPSRC (EP/I014462/1), and the European Research Council (ERC) under the European Union's Horizon 2020 research and innovation program (Grant Agreements No. 682399 and No. 653782).

[1] T. Tajima and J. M. Dawson, Phys. Rev. Lett. 43, 267 (1979).

[2] S. P. D. Mangles, C. D. Murphy, Z. Najmudin, A. G. R. Thomas et al., Nature (London) 431, 535 (2004).

[3] C. G. R. Geddes, C. Toth, J. van Tilborg, E. Esarey, C. B. Schroeder, D. Bruhwiler, C. Nieter, J. Cary, and W. P. Leemans, Nature (London) 431, 538 (2004).

[4] J. Faure, Y. Glinec, A. Pukhov, S. Kiselev, S. Gordienko, E. Lefebvre, J. P. Rousseau, F. Burgy, and V. Malka, Nature (London) 431, 541 (2004).

[5] O. Lundh, J. Lim, C. Rechatin, L. Ammoura, A. BenIsmail, X. Davoine, G. Gallot, J.-P. Goddet, E. Lefebvre, V. Malka, and J. Faure, Nat. Phys. 7, 219 (2011).

[6] W. P. Leemans, A. J. Gonsalves, H. S. Mao, K. Nakamura, C. Benedetti, C. B. Schroeder, C. Tóth, J. Daniels, D. E. Mittelberger, S. S. Bulanov, J. L. Vay, C. G. R. Geddes, and E. Esarey, Phys. Rev. Lett. 113, 245002 (2014).

[7] X. Wang, R. Zgadzaj, N. Fazel, Z. Li et al., Nat. Commun. 4, 1988 (2013).

[8] H. T. Kim, K. H. Pae, H. J. Cha, I. J. Kim, T. J. Yu, J. H. Sung, S. K. Lee, T. M. Jeong, and J. Lee, Phys. Rev. Lett. 111, 165002 (2013).

[9] A. Rousse, K. T. Phuoc, R. C. Shah, A. Pukhov, E. Lefebvre, V. Malka, S. Kiselev, F. Burgy, J. P. Rousseau, D. P. Umstadter, and D. Hulin, Phys. Rev. Lett. 93, 135005 (2004).

[10] S. Kneip, C. McGuffey, J. L. Martins, S. F. Martins et al., Nat. Phys. 6, 980 (2010). 
[11] F. Albert, A. G. R. Thomas, S. P. D. Mangles, S. Banerjee, S. Corde, A. Flacco, M. Litos, D. Neely, J. Vieira, Z. Najmudin, R. Bingham, C. Joshi, and T. Katsouleas, Plasma Phys. Controlled Fusion 56, 084015 (2014).

[12] A. Pukhov and J. Meyer-ter Vehn, Appl. Phys. B 74, 355 (2002).

[13] S. Kneip, S. R. Nagel, C. Bellei, N. Bourgeois et al., Phys. Rev. Lett. 100, 105006 (2008).

[14] S. P. D. Mangles, G. Genoud, M. S. Bloom, M. Burza, Z. Najmudin, A. Persson, K. Svensson, A. G. R. Thomas, and C. G. Wahlström, Phys. Rev. Accel. Beams 15, 011302 (2012).

[15] This definition of $E_{\mathrm{c}}$ is different from that used in Refs. [13,16] but consistent with Refs. [9,17,18].

[16] E. Esarey, B. A. Shadwick, P. Catravas, and W. P. Leemans, Phys. Rev. E 65, 056505 (2002).

[17] A. Rousse, K. T. Phuoc, R. C. Shah, R. Fitour, and F. Albert, Eur. Phys. J. D 45, 391 (2007).

[18] J. D. Jackson, Classical Electrodynamics, 3rd ed. (Wiley, New York, 1999).

[19] C. M. Huntington, A. G. R. Thomas, C. McGuffey, T. Matsuoka, V. Chvykov, G. Kalintchenko, S. Kneip, Z. Najmudin, C. Palmer, V. Yanovsky, A. Maksimchuk, R. P. Drake, T. Katsouleas, and K. Krushelnick, Phys. Rev. Lett. 106, 105001 (2011).

[20] S. P. D. Mangles, A. G. R. Thomas, M. C. Kaluza, O. Lundh, F. Lindau, A. Persson, F. S. Tsung, Z. Najmudin, W. B. Mori, C. G. Wahlström, and K. Krushelnick, Phys. Rev. Lett. 96, 215001 (2006).

[21] S. Cipiccia, M. R. Islam, B. Ersfeld, R. P. Shanks et al., Nat. Phys. 7, 867 (2011).

[22] W. Lu, M. Tzoufras, C. Joshi, F. S. Tsung, W. B. Mori, J. Vieira, R. Fonseca, and L. Silva, Phys. Rev. Accel. Beams 10, 061301 (2007).

[23] J. Schreiber, C. Bellei, S. P. D. Mangles, C. Kamperidis, S. Kneip, S. R. Nagel, C. A. J. Palmer, P. P. Rajeev, M. J. V. Streeter, and Z. Najmudin, Phys. Rev. Lett. 105, 235003 (2010).
[24] W. Mori, IEEE J. Quantum Electron. 33, 1942 (1997).

[25] I. Y. Kostyukov, A. Pukhov, and S. Kiselev, Phys. Plasmas 11, 5256 (2004).

[26] A. G. R. Thomas, Phys. Plasmas 17, 056708 (2010).

[27] M. J. V. Streeter, S. Kneip, M. S. Bloom, R. A. Bendoyro, O. Chekhlov, A. E. Dangor, A. Döpp, C. J. Hooker, J. Holloway, J. Jiang, N. C. Lopes, H. Nakamura, P. A. Norreys, C. A. J. Palmer, P. P. Rajeev, J. Schreiber, D. R. Symes, M. Wing, S. P. D. Mangles, and Z. Najmudin, Phys. Rev. Lett. 120, 254801 (2018).

[28] S. Kneip, Z. Najmudin, and A. G. R. Thomas, High Energy Density Phys. 8, 133 (2012).

[29] S. P. D. Mangles, G. Genoud, S. Kneip, M. Burza, K. Cassou, B. Cros, N. P. Dover, C. Kamperidis, Z. Najmudin, A. Persson, J. Schreiber, F. Wojda, and C. G. Wahlström, Appl. Phys. Lett. 95, 181106 (2009).

[30] M. Schnell, A. Sävert, B. Landgraf, M. Reuter, M. Nicolai, O. Jäckel, C. Peth, T. Thiele, O. Jansen, A. Pukhov, O. Willi, M. C. Kaluza, and C. Spielmann, Phys. Rev. Lett. 108, 075001 (2012).

[31] J. Wenz, S. Schleede, K. Khrennikov, M. Bech, P. Thibault, M. Heigoldt, F. Pfeiffer, and S. Karsch, Nat. Commun. 6, 7568 (2015).

[32] S. Fourmaux, S. Corde, K. T. Phuoc, P. M. Leguay, S. Payeur, P. Lassonde, S. Gnedyuk, G. Lebrun, C. Fourment, V. Malka, S. Sebban, A. Rousse, and J. C. Kieffer, New J. Phys. 13, 033017 (2011).

[33] F. Albert, B. B. Pollock, J. L. Shaw, K. A. Marsh, J. E. Ralph, Y.H. Chen, D. Alessi, A. Pak, C. E. Clayton, S. H. Glenzer, and C. Joshi, Phys. Rev. Lett. 111, 235004 (2013).

[34] J. M. Cole, J. C. Wood, N. C. Lopes, K. Poder, R. L. Abel, S. Alatabi, J. S. J. Bryant, A. Jin, S. Kneip, K. Mecseki, D. R. Symes, S. P. D. Mangles, and Z. Najmudin, Sci. Rep. 5, 13244 (2015).

[35] S. P. D. Mangles, CERN Yellow Rep. 1, 289 (2016).

[36] https://doi.org/10.5281/zenodo.3765331. 